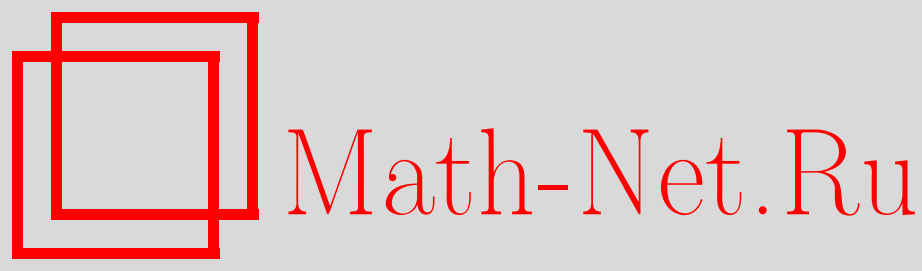

А. А. Хамзин, Р. Р. Нигматуллин, Форма линии ЯМР системы ядерных спинов с равными константами спинспиновых взаимодействий, ТМФ, 2011, том 167, номер 1, 111-122

DOI: https://doi.org/10.4213/tmf6628

Использование Общероссийского математического портала Math-Net.Ru подразумевает, что вы прочитали и согласны с пользовательским соглашением http: //www . mathnet.ru/rus/agreement

Параметры загрузки:

IP : 3.81 .55 .215

26 апреля 2023 г., 08:04:10

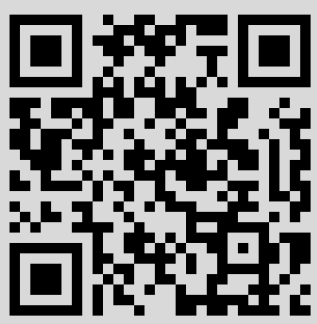




\section{ФОРМА ЛИНИИ ЯМР СИСТЕМЫ ЯДЕРНЫХ СПИНОВ С РАВНЫМИ КОНСТАНТАМИ СПИН-СПИНОВЫХ ВЗАИМОДЕЙСТВИЙ}

Получено выражение для линии ЯМР при низких температурах для системы ядерных спинов, описываемой гамильтонианом с равными константами спин-спиновых взаимодействий. Показано, что в случае анизотропии “легкая ось" линия имеет логарифмическую низкочастотную сингулярность и экспоненциально убывающую высокочастотную асимптотику при температуре аномального пика теплоемкости. В случае анизотропии “легкая плоскость" линия имеет традиционную гауссову форму. Обсуждена возможность обнаружения специфических термодинамических и магнитных свойств рассматриваемой модельной системы по данным ЯМР.

Ключевые слова: спин-спиновое взаимодействие, спад свободной индукции, линия ЯМР, магнитные упорядоченные структуры.

\section{1. ВВЕДЕНИЕ}

Современные методы ядерного магнитного резонанса (ЯМР) широко используются в физико-химических исследованиях [1]-[3]. Ядерные спины играют роль "меток", позволяющих выявить пространственное расположение ядер и их взаимодействие с окружением, что дает возможность решать задачи определения внутренней структуры исследуемых веществ и изучать различные динамические процессы. Благодаря значительным успехам в термодинамике спиновых систем, криогенной технике, технике динамической поляризации ядер и теории ЯМР появилась возможность осуществлять и наблюдать различные типы спиновых упорядоченных структур [4], [5]. Изменение спиновой конфигурации определяет характер формы линии ЯМР, ширина которой дает информацию о температуре перехода между различными спиновыми структурами.

В последнее время в связи с достижениями в области нанотехнологий усилился интерес к изучению конечных спиновых систем (нанокластеров). Рост интереса

*Казанский государственный энергетический университет, Казань, Россия. E-mail: airat.khamzin@rambler.ru

${ }^{\dagger}$ Казанский (Приволжский) федеральный университет, Казань, Россия. E-mail: nigmat@knet.ru 
к объектам с ограниченным числом спиновых моментов - молекулярным магнетикам, наноструктурам и нанокластерам - вызывает необходимость разработки точных аналитических методов исследований. При теоретическом описании магнитоактивных кластеров возникает проблема формулировки корректной и адекватной модели, которая качественно и количественно могла бы претендовать на описание магнитных и термодинамических свойств наномагнетиков. При этом желательно, чтобы модель допускала аналитическое решение.

В работе [6] авторами была предложена упрощенная модель описания свойств спиновых систем в мезоскопических магнитоактивных кластерах, получившая название модели равных спин-спиновых взаимодействий (РССВ), которая допускает точное аналитическое рассмотрение. В работе [7] подробно изучалась термодинамика данной модели. Анализ термодинамических свойств модели РССВ выявил ряд их специфических особенностей. В частности, было показано, что в конечной спиновой системе, описываемой моделью РССВ, образуются топологические возбуждения (солитоны), которые, во-первых, обусловливают отсутствие дальнего порядка в системе, а во-вторых, формируют аномальное поведение теплоемкости при низких температурах (появление дополнительного низкотемпературного пика). Аномальное поведение теплоемкости модели связывается с возникновением в рассматриваемой системе спиновой щели, наличие которой в спектре магнитных возбуждений приводит также к специфическим особенностям магнитных свойств модели [8], а именно к немонотонному поведению восприимчивости и наличию дробных плато в полевой зависимости намагниченности.

Поскольку модель РССВ предлагается авторами для описания конкретных физических свойств спиновых систем, то естественно возникает вопрос: как экспериментально провести непосредственную проверку полученных специфических свойств? Метод ЯМР ввиду высокой разрешающей способности спектров является наиболее приемлемым инструментом для проверки предсказанных специфических особенностей термодинамических и магнитных свойств спиновых систем, которые могут быть описаны моделью РССВ. Поэтому в настоящей работе авторами ставится задача построения метода аналитического описания линии ЯМР при низких температурах спиновых систем с равными константами спин-спиновых взаимодействий для выявления зависимости спектров ЯМР от спиновой конфигурации рассматриваемой физической системы и характерных параметров (например, от температуры перехода в фазу аномального поведения теплоемкости и величины спиновой щели).

Структура настоящей статьи следующая. В разделе 2 мы формулируем модель РССВ и проводим аргументы в пользу ее применимости для описания физических свойств конечных спиновых систем. В разделе 3 приводится расчет спада свободной индукции (ССИ) и линии ЯМР для низких температур. В заключении делаются выводы из проведенных расчетов.

\section{2. ФОРМУЛИРОВКА МОДЕЛИ}

Рассмотрим систему из $N$ взаимодействующих частиц, несущих ядерный спин $1 / 2$ и заключенных в некоторый конечный объем $V$ (потенциальную яму). Гамильтониан рассматриваемой системы, который зависит от времени, запишем в традиционной 
форме Гейзенберга:

$$
H(t)=-\omega \sum_{f=1}^{N} I_{f}^{z}-\frac{1}{2} \sum_{f, g=1}^{N}\left(\frac{1}{2} U_{f, g}^{+-}(t)\left(I_{f}^{+} I_{g}^{-}+I_{f}^{-} I_{g}^{+}\right)+U_{f, g}^{z z}(t) I_{f}^{z} I_{g}^{z}\right)
$$

где $I_{f}^{\alpha}, \alpha=x, y, z, I_{f}^{ \pm}=I_{f}^{x} \pm i I_{f}^{y}$ - компоненты оператора ядерного спина; $U_{f, g}^{\alpha \beta}(t)-$ компоненты зависящего от времени потенциала двухчастичного спин-спинового взаимодействия; $\omega$ - зеемановская частота ядерных спинов в магнитном поле $B, \omega=$ $\gamma B$, направленного вдоль оси $z$. Под действием конкретных физических факторов потенциал взаимодействия может изменяться как по величине, так и по знаку в течение характерного времени $\tau_{1}$. Естественно, что в рассматриваемой системе существует другой масштаб времени - характерное время $\tau_{2}$ переворота намагниченности спина частицы вследствие взаимодействия с ближайшим соседним спином (совпадающее со временем флип-флоп-перехода). Если в системе реализуется ситуация $\tau_{1} \ll \tau_{2}$, то спин-спиновое взаимодействие частиц будет определяться не их мгновенным расположением, а усредненным распределением частиц. Наличие в системе малого адиабатического параметра $\varepsilon=\tau_{1} / \tau_{2} \ll 1$ позволяет определить усредненный спиновый гамильтониан, управляющий поведением спинов на интервале времени $\Delta t$, подчиняющемся условию $\tau_{1} \ll \Delta t \ll \tau_{2}$. Усредняя точный гамильтониан $H$ по времени $\Delta t$ в нулевом порядке по параметру $\varepsilon$, получим средний (эффективный) гамильтониан

$$
\begin{gathered}
\bar{H}=-\omega \sum_{f=1}^{N} I_{f}^{z}-\frac{1}{2} \sum_{f, g=1}^{N}\left(\frac{1}{2} \overline{U_{f, g}^{+-}}\left(I_{f}^{+} I_{g}^{-}+I_{f}^{-} I_{g}^{+}\right)+\overline{U_{f, g}^{z z}} I_{f}^{z} I_{g}^{z}\right) \\
\overline{U_{f, g}^{\alpha \beta}}=\frac{1}{\Delta t} \int_{0}^{\Delta t} U_{f, g}^{\alpha \beta}\left(r_{f}\left(t^{\prime}\right), r_{g}\left(t^{\prime}\right)\right) d t^{\prime}
\end{gathered}
$$

с точностью $O\left(\varepsilon^{1}\right)$. Следующий решающий шаг состоит в замене интегрирования по времени на интегрирование по пространственным координатам в ограниченном объеме конфигурационного пространства. Эквивалентность временно́го и пространственного усреднений опирается на эргодическую гипотезу

$$
\frac{\delta t\left(d r^{N}, d p^{N}\right)}{t}=Q^{-1} e^{-E / T} d r^{N} d p^{N}
$$

где введенная система обозначений предполагает, что рассматриваемая точка, лежащая в фазовом пространстве $r^{N}-p^{N}$, при движении во временно́м интервале $t$, $\tau_{1} \ll t \ll \tau_{2}$, тратит только определенную часть, а именно $Q^{-1} e^{-E / T} d r^{N} d p^{N}$, полного времени $t$ в объеме $d r^{N} d p^{N}$. Здесь $E-$ полная энергия.

Введем в рассмотрение равновесную парную функцию распределения для частиц 1 и 2 :

$$
D_{2}\left(r_{1}, r_{2}\right)=\frac{\int_{V} d^{3} r_{3} \ldots \int_{V} d^{3} r_{N} e^{-U\left(r^{N}\right) / T}}{\int_{V} d^{3} r_{1} \ldots \int_{V} d^{3} r_{N} e^{-U\left(r^{N}\right) / T}},
$$

где $U\left(r^{N}\right)$ - межчастичные электростатические взаимодействия. Принимая во внимание условие эргодичности (1), найдем, что поведение спиновых степеней свободы 
на временно́м масштабе $\Delta t$ определяется статическим эффективным гамильтониаHOM

$$
\begin{aligned}
\bar{H} & =-\omega \sum_{f} I_{f}^{z}-\frac{A_{\|}}{2} \sum_{f, g} I_{f}^{z} I_{g}^{z}-\frac{A_{\perp}}{4} \sum_{f, g}\left(I_{f}^{+} I_{g}^{-}+I_{g}^{-} I_{f}^{+}\right)= \\
& =-\omega I^{z}-\frac{1}{2}\left(A_{\|}-A_{\perp}\right)\left(I^{z}\right)^{2}-\frac{A_{\perp}}{2} I^{2}+\frac{N}{8}\left(A_{\|}+2 A_{\perp}\right),
\end{aligned}
$$

где пространственно-независимые константы парных взаимодействий $A_{\|}$и $A_{\perp}$ для любой пары спинов $f$ и $g$ определяются выражениями

$$
\begin{aligned}
& A_{\|}=\int_{V} \int_{V} U_{f, g}^{z z}\left(r_{f}, r_{g}\right) D_{2}\left(r_{f}, r_{g}\right) d^{3} r_{f} d^{3} r_{g}, \\
& A_{\perp}=\int_{V} \int_{V} U_{f, g}^{+-}\left(r_{f}, r_{g}\right) D_{2}\left(r_{f}, r_{g}\right) d^{3} r_{f} d^{3} r_{g},
\end{aligned}
$$

где $I^{z}=\sum_{f=1}^{N} I_{f}^{z}, I^{ \pm}=\sum_{f=1}^{N} I_{f}^{ \pm}-$компоненты полного спина $\vec{I}, I^{2}-$ квадрат величины полного спина, который определяется выражением

$$
I^{2}=\frac{I^{+} I^{-}+I^{-} I^{+}}{2}+\left(I^{z}\right)^{2}
$$

В качестве примера спиновой многочастичной системы, в которой реализуется описанный выше механизм усреднения спин-спиновых взаимодействий, рассмотрим систему спин-несущих молекул газа в нанополости [9], [10].

Действительно, пусть рассматривается нанополость, заполненная газом с $N$ спиннесущими молекулами (спин равен $1 / 2$ ) во внешнем магнитном поле $B \| Z$, где $Z$ одна из координатных осей (см. рис. 1). Зависящий от времени точный дипольный гамильтониан данной системы может быть записан в виде [10]

$$
H(t)=\omega \sum_{n=1}^{N} I_{n z}+\sum_{1 \leqslant i \leqslant j}^{N} h_{i, j}(t), \quad h_{i, j}(t)=\gamma^{2} \hbar P_{2}\left(\cos \theta_{i j}(t)\right) r_{i j}^{-3}\left(\vec{I}_{i} \vec{I}_{j}-3 I_{i}^{z} I_{j}^{z}\right)
$$

где $\theta_{i j}$ - полярный угол между вектором $\vec{r}_{i j}$ и внешним магнитным полем $B$. В рассматриваемой системе действительно существуют два сильно различающихся масштаба времени: характерное время пролета спин-несущей молекулы газа между двумя последовательными соударениями со стенкой нанополости $\tau_{1} \approx 10^{-11}$ с и характерное время переворота намагниченности спина выделенного ядра из-за его дипольного взаимодействия с ближайшим соседним спином $\tau_{2} \approx 10^{-4} \mathrm{c}, \tau_{2} \gg \tau_{1}$. Это неравенство означает, что в течение времени $\tau_{2}$ выделенное ядро успевает пролететь между стенками нанополости $\tau_{2} / \tau_{1}=10^{7}$ раз (существует малый адиабатический параметр $\left.\varepsilon=10^{-7} \ll 1\right)$, и, следовательно, диполь-дипольное взаимодействие выделенного ядра с соседним ядром определяется не мгновенным расположением, а усредненным распределением ядер по всему объему нанополости. Проводя усреднение точного гамильтониана (4) по алгоритму, описанному выше, приходим к эффективному гамильтониану

$$
\bar{H}=\omega I_{z}+\frac{D}{2}\left((\zeta+1) I_{z}^{2}-I^{2}\right)
$$




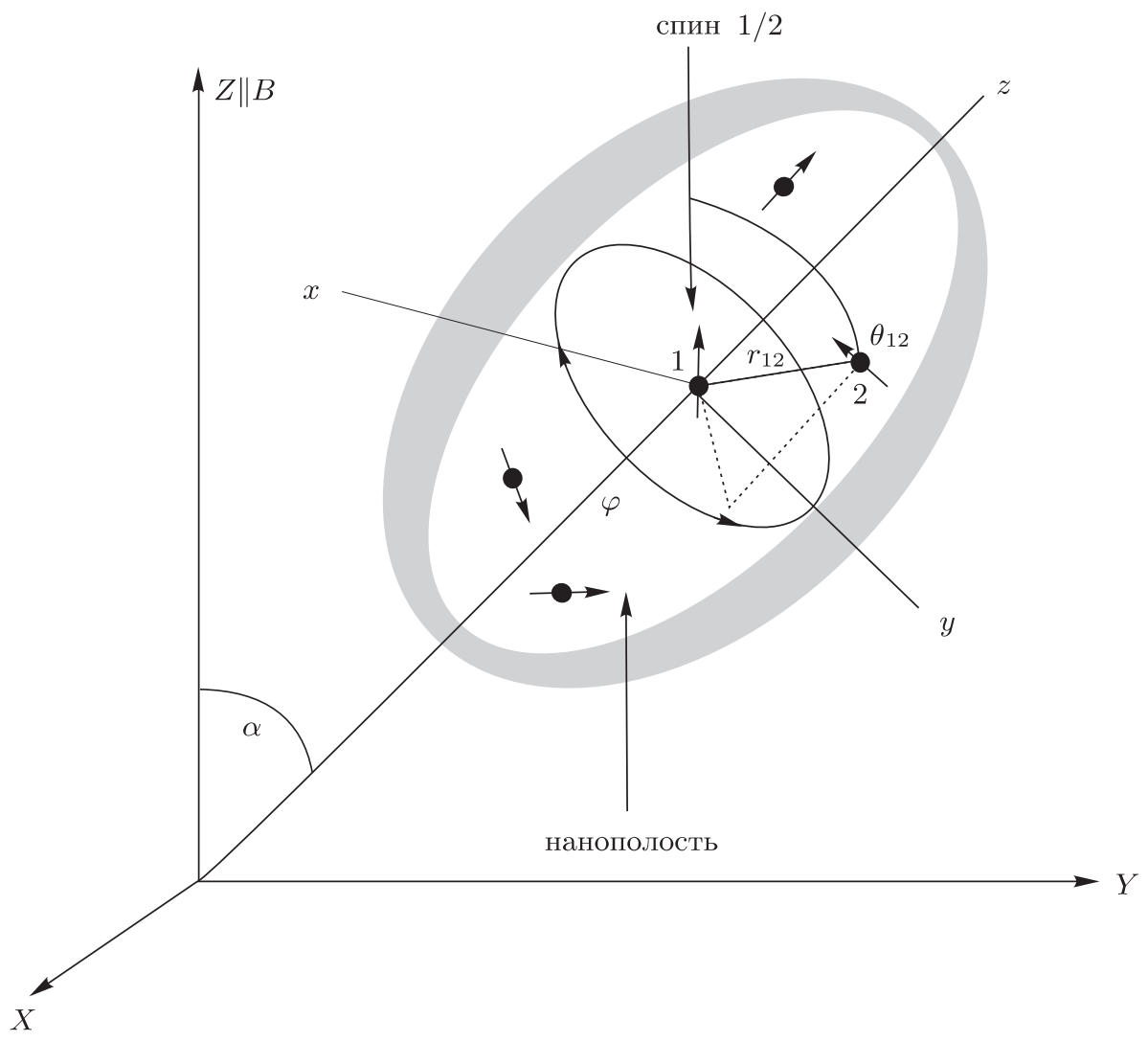

Рис. 1. Нанополость, заполненная спин-несущими молекулами, совершающими быстрое тепловое движение во внешнем магнитном поле.

в котором константа дипольной связи любой пары спинов находится по формуле (3) и равна [10]

$$
D=\gamma^{2} h P_{2}(\cos \alpha) \frac{f}{V}
$$

Здесь угол $\alpha$ определяет ориентацию главной оси эллипсоидальной полости по отношению к внешнему полю $B$ (см. рис. 1$), V$ - объем полости, $f$ - формфактор, который является монотонной функцией отношения главных осей эллипсоидальной полости $a / b$ со следующими предельными значениями [10]: $f=1 / 3$ (полый цилиндр, $a / b \gg 1), f=0$ (полый шар, $a / b=1), f=-2 / 3$ (полый диск, $a / b \ll 1$ ). Таким образом, диполь-дипольные взаимодействия ядерных спинов в спин-несущих молекулах газа в несферических нанопорах также усредняются за счет быстрой молекулярной диффузии, но не полностью (пространственный конфайнмент). Остаточные (усредненные) диполь-дипольные взаимодействия описываются одной и той же константой для всех пар ядерных спинов. В результате появляется возможность нахождения точных решений в задачах о переносе поляризации в спиновой системе в нанопоpe [10], [11] и о форме линии ЯМР [12]. 
Модель, определяемая гамильтонианом (2), получила название модели РССВ [6]. Переход к гамильтониану (2) значительно упрощает анализ термодинамических [7] и магнитных [8] свойств конечных спиновых систем. Основным достоинством этой модели является возможность получения точного решения для динамики многоспиновой системы при любом $N$ [6], [10], [11] и строгого аналитического описания формы линии ЯМР как для высоких [12], так и для низких температур, что будет показано ниже.

\section{3. ФОРМА ЛИНИИ ЯМР ПРИ НИЗКИХ ТЕМПЕРАТУРАХ}

Форма линии ЯМР определяется как фурье-преобразование ССИ, который при конечных температурах имеет вид нормированной корреляционной функции [13], [14]:

$$
G(t)=\frac{\left\langle I^{+}(t) I^{-}\right\rangle}{\left\langle I^{+} I^{-}\right\rangle}
$$

где $\langle\ldots\rangle=\operatorname{Sp}\left(\ldots e^{-\beta \bar{H}}\right) / \mathrm{Sp}\left(e^{-\beta \bar{H}}\right)$ означает термодинамическое усреднение по равновесному статистическому ансамблю, $\beta=1 / T, T$ - температура, а временна́я зависимость оператора $I^{+}(t)=e^{i t \bar{H}} I^{+} e^{-i t \bar{H}}$ в представлении Гейзенберга легко определяется из уравнения движения

$$
\frac{d I^{+}(t)}{d t}=i\left[\bar{H}, I^{+}\right]=-i\left(\omega+\left(A_{\|}-A_{\perp}\right)\left(I^{z}-1 / 2\right)\right) I^{+}
$$

и имеет вид

$$
I^{+}(t)=e^{-i\left(\omega+\left(A_{\|}-A_{\perp}\right)\left(I^{z}-1 / 2\right)\right) t} I^{+}(0) .
$$

Чтобы рассчитать ССИ $G(t)$, представим его в виде $G(t)=R(t) / R(0)$, где $R(t)=$ $\operatorname{Sp}\left(I^{+}(t) I^{-} e^{-\beta \bar{H}}\right)$. Поскольку собственные значения гамильтониана (2) известны:

$$
E_{m s}=-\omega m-\frac{A_{\perp}}{2} \delta m^{2}-\frac{A_{\perp}}{2} s(s+1)+\frac{N A_{\perp}}{8}(3+\delta),
$$

где $s=s_{\min }, \ldots, N / 2\left(s_{\min }=1 / 2\right.$ для нечетного и $s_{\min }=0$ для четного количества частиц $N$ в системе), $m=-s, \ldots, s, \delta=A_{\|} / A_{\perp}-1$ - параметр анизотропии $(\delta<0-$ анизотропия "легкая плоскость", $\delta>0$ - анизотропия "легкая ось"), то функция $R(t)$ выражается в виде

$$
R(t)=\sum_{s=s_{\min }}^{N / 2} g(N, s) e^{\beta A_{\perp} s(s+1) / 2} \sum_{m=-s}^{s}(s+m)(s-m+1) e^{\operatorname{sgn}(\delta) 2 i \tau(1 / 2-m)+\beta A_{\perp} \delta m^{2} / 2} .
$$

Здесь фактор

$$
g(N, s)=\frac{2 s+1}{N+1}\left(\begin{array}{c}
N+1 \\
N / 2-s
\end{array}\right)
$$

определяет число способов группировки $N$ спинов $1 / 2$ в полный спин $s, \tau=t|\delta| A_{\perp} / 2$, $A_{\perp}>0$.

Точный расчет суммы (5) затруднен из-за наличия слагаемых, квадратичных по $s$ и $m$ в показателях экспонент. Эту трудность в какой-то степени можно обойти, если воспользоваться тождеством

$$
e^{\mp k^{2} / a}=\frac{1}{2} \sqrt{\frac{a}{\pi}} \int_{-\infty}^{\infty} e^{-a x^{2} / 4-i \varepsilon k x} d x, \quad a>0, \quad \varepsilon=\left\{\begin{array}{l}
1 \\
i
\end{array}\right\} .
$$


Применяя тождество (6) и выбирая верхний знак для анизотропии “легкая плоскость" $(\delta<0)$ и нижний для анизотропии “легкая ось” $(\delta>0)$, из выражения $(5)$ получим

$$
\begin{aligned}
R(t)= & \sqrt{\frac{T}{2 \pi A_{\perp}|\delta|}} e^{\mp i \tau} \int_{-\infty}^{\infty} e^{-T x^{2} /\left(2 A_{\perp}|\delta|\right)} d x \sum_{s=s_{\min }}^{N / 2} g(N, s) e^{\beta A_{\perp} s(s+1) / 2} \times \\
& \times \sum_{m=-s}^{s}(s+m)(s-m+1) e^{-i m z},
\end{aligned}
$$

где $z=\varepsilon x \mp 2 \tau$. После такой "линеаризации" показателя экспоненты можно произвести суммирование по $m$ :

$$
\sum_{m=-s}^{s}(s+m)(s-m+1) e^{-i m z}=\frac{1}{2} e^{-i z / 2} \frac{(s+1) \sin (s z)-s \sin ((s+1) z)}{\sin ^{3}(z / 2)} .
$$

В результате выражение (7) примет вид

$$
\begin{aligned}
R(t)=\frac{1}{2} & \sqrt{\frac{T}{2 \pi A_{\perp}|\delta|}} \int_{-\infty}^{\infty} e^{-T x^{2} /\left(2 A_{\perp}|\delta|\right)-i \varepsilon x / 2} d x \times \\
& \times \sum_{s=s_{\text {min }}}^{N / 2} g(N, s)\left(\frac{(s+1) \sin (s z)-s \sin ((s+1) z)}{\sin ^{3}(z / 2)}\right) e^{A_{\perp} s(s+1) /(2 T)} .
\end{aligned}
$$

Для облегчения суммирования по $s$ положим в показателе экспоненты суммируемого выражения $s \simeq N / 2$, предполагая, что рассматриваются низкие температуры. Тогда суммирование в формуле (8) проводится точно, и мы получаем следующее выражение для $R(t)$ :

$$
\begin{gathered}
R(t)=N 2^{N-1} \sqrt{\frac{T}{2 \pi A_{\perp}|\delta|}} e^{A_{\perp} N(N+2) /(8 T)} W(\tau), \\
W(\tau)=\int_{-\infty}^{\infty} e^{-T x^{2} /\left(2 A_{\perp}|\delta|\right)-i \varepsilon x / 2}\left(\cos \frac{z}{2}\right)^{N-1} d x,
\end{gathered}
$$

где использовалось тождество

$$
\sum_{s=0}^{n} g(2 n, s)\left(\frac{(s+1) \sin (s z)-s \sin ((s+1) z)}{\sin ^{3}(z / 2)}\right)=n 2^{2 n+1} \cos ^{2 n-1}\left(\frac{z}{2}\right) .
$$

Осуществим асимптотическую оценку интеграла (9) при $N \gg 1$, воспользовавшись методом Лапласа [15]. Представим интеграл $W(\tau)(9)$ в виде

$$
W(\tau)=\int_{-\infty}^{\infty} e^{N \Phi(x, \tau)} d x, \quad \Phi(x, \tau)=\ln \cos \left(\frac{\varepsilon x \mp 2 \tau}{2}\right)-\frac{T x^{2}}{8 T_{0}}-\frac{i \varepsilon x}{2 N},
$$

где $T_{0}=N A_{\perp}|\delta| / 4$. Отметим, что температура $T_{0}$ определяет положение аномального пика теплоемкости при анизотропии “легкая ось" [7], а также величину спиновой щели $\Delta=2 T_{0}$. 
Поскольку нас интересует только амплитуда ССИ $|G(t)|$, то проведем лишь оценкy

$$
|W(\tau)| \leqslant \int_{-\infty}^{\infty} e^{N \operatorname{Re} \Phi(x, \tau)} d x
$$

где

$$
\operatorname{Re} \Phi(x, \tau) \equiv S(x, \tau)= \begin{cases}\ln \cos \left(\frac{x}{2}-\tau\right)-\frac{T}{8 T_{0}} x^{2}, & \delta<0 \\ \frac{1}{2} \ln \left(\operatorname{sh}^{2}\left(\frac{x}{2}\right)+\cos ^{2} \tau\right)-\frac{T}{8 T_{0}} x^{2}+\frac{x}{2 N}, & \delta>0 .\end{cases}
$$

В соответствии с методом Лапласа находим уравнение для стационарной точки $x^{*}$ :

$$
S_{x}^{\prime}(x, \tau)=0 \Rightarrow \begin{cases}\operatorname{tg}\left(\frac{x^{*}}{2}-\tau\right)=-\frac{T}{2 T_{0}} x^{*}, & \delta<0 \\ \frac{\operatorname{sh} x^{*}}{\operatorname{sh}^{2}\left(x^{*} / 2\right)+\cos ^{2} \tau} \cong \frac{T}{T_{0}} x^{*}, \quad \delta>0 .\end{cases}
$$

Приближенное решение уравнений (11) имеет вид

$$
x^{*}(\tau)=\frac{2 \tau}{1+T / T_{0}}
$$

при $\delta<0$ и

$$
x^{*}(\tau)= \begin{cases}0, & T>T_{0}\left(1+\tau^{2}\right), \\ \pm \sqrt{\frac{12\left(1+\tau^{2}-T / T_{0}\right)}{1+4 \tau^{2}},}, & T \leqslant T_{0}\left(1+\tau^{2}\right),\end{cases}
$$

при $\delta>0$. Решение (13) напоминает решение для спонтанной намагниченности в среднеполевой теории ферромагнетика. Это совпадение не случайно, поскольку решение рассматриваемой здесь модели в термодинамическом пределе $(N \rightarrow \infty$, $V \rightarrow \infty, N / V=$ const) совпадает с результатами теории молекулярного поля Вейсca [16], [17].

Согласно методу Лапласа [15] асимптотическая оценка интеграла (10) при $N \gg 1$ имеет вид

$$
|W(\tau)| \approx \sqrt{\frac{-2 \pi}{N S_{x x}^{\prime \prime}\left(x^{*}(\tau), \tau\right)}} e^{N S\left(x^{*}(\tau), \tau\right)}\left(1+O\left(\frac{1}{N}\right)\right),
$$

следовательно, оценка ССИ примет вид

$$
|G(t)| \approx \frac{e^{N\left(S\left(x^{*}(\tau), \tau\right)-S\left(x^{*}(0), 0\right)\right)}}{\sqrt{S_{x x}^{\prime \prime}\left(x^{*}(\tau), \tau\right) / S_{x x}^{\prime \prime}\left(x^{*}(0), 0\right)}} .
$$

В случае анизотропии типа "легкая плоскость" $(\delta<0)$ для $\tau \ll 1$ имеем

$$
\begin{gathered}
S\left(x^{*}(\tau), \tau\right)-S\left(x^{*}(0), 0\right) \approx-\frac{\tau^{2}}{2\left(1+T_{0} / T\right)}, \\
\frac{S_{x x}^{\prime \prime}\left(x^{*}(\tau), \tau\right)}{S_{x x}^{\prime \prime}\left(x^{*}(0), 0\right)} \approx 1+\frac{T_{0}}{T} \frac{\tau^{2}}{\left(1+T_{0} / T\right)^{3}}, \\
|G(t)| \approx \\
\left(1+\frac{T_{0}}{T} \frac{\tau^{2}}{\left(1+T_{0} / T\right)^{3}}\right)^{-1 / 2} e^{-(N / 2) \tau^{2} /\left(1+T_{0} / T\right)} .
\end{gathered}
$$


В случае анизотропии типа “легкая ось" $(\delta>0)$ при $\tau \ll 1$ получим

$$
\begin{aligned}
S\left(x^{*}(\tau), \tau\right)-S\left(x^{*}(0), 0\right) & \approx \begin{cases}-\frac{\tau^{2}}{2}, & T>T_{0}\left(1+\tau^{2}\right), \\
-\frac{1}{2} \psi(\kappa) \tau^{2}, & T \leqslant T_{0}\left(1+\tau^{2}\right),\end{cases} \\
\frac{S_{x x}^{\prime \prime}\left(x^{*}(\tau), \tau\right)}{S_{x x}^{\prime \prime}\left(x^{*}(0), 0\right)} & \approx \begin{cases}1-\frac{\tau^{2}}{T / T_{0}-1}, & T>T_{0}\left(1+\tau^{2}\right), \\
1+\varphi(\kappa) \tau^{2}, & T \leqslant T_{0}\left(1+\tau^{2}\right),\end{cases}
\end{aligned}
$$

где

$$
\begin{gathered}
\psi(\kappa)=1-\operatorname{th}^{2}(\sqrt{3} \kappa)+\frac{1-4 \kappa^{2}}{\kappa}\left[3 \kappa\left(1-\kappa^{2}\right)-\sqrt{3} \operatorname{th}(\sqrt{3} \kappa)\right] \approx 1-3 \kappa^{2}, \\
\varphi(\kappa)=\frac{1-\operatorname{th}^{2}(\sqrt{3} \kappa)}{\kappa} \frac{2 \kappa(2-\operatorname{ch}(2 \sqrt{3} \kappa))-\sqrt{3}\left(1-4 \kappa^{2}\right) \operatorname{sh}(2 \sqrt{3} \kappa)}{1+\kappa^{2}-\left(1-\kappa^{2}\right) \operatorname{ch}(2 \sqrt{3} \kappa)} \approx \frac{1}{\kappa^{2}}-3-\frac{3}{10} \kappa^{2},
\end{gathered}
$$

$\kappa=\sqrt{1-T / T_{0}}$. Здесь мы приводим также приближенные выражения для функций $\psi(\kappa), \varphi(\kappa)$ вблизи температуры $T_{0}$. Амплитуда ССИ в рассматриваемом случае анизотропии примет вид

$$
|G(t)| \approx \begin{cases}\frac{1}{\sqrt{1-\tau^{2} /\left(T / T_{0}-1\right)}} e^{-N \tau^{2} / 2}, & T>T_{0}\left(1+\tau^{2}\right), \\ \frac{1}{\sqrt{1+\varphi(\kappa) \tau^{2}}} e^{-N \psi(\kappa) \tau^{2} / 2}, & T \leqslant T_{0}\left(1+\tau^{2}\right) .\end{cases}
$$

Из формулы (15) можно видеть, что при $T \rightarrow T_{0}-0$ происходит замедление ССИ:

$$
|G(t)| \approx \frac{1}{\sqrt{1+\tau^{2} / \kappa^{2}}} e^{-N \tau^{2} / 2} \leqslant \frac{1}{\sqrt{1+\tau^{2} / \kappa^{2}}} .
$$

Это очевидно связано с возникновением в рассматриваемой спиновой системе комплексов с долгоживущими поляризациями. На рис. 2 приведен график зависимости ССИ от времени и температуры при $\delta>0$, из которого отчетливо видно, как происходит переход при $T=T_{0}$ от быстрого гауссова спада $G(t)$ к более медленному степенно́му $(\sim 1 / t)$.

Линия ЯМР

$$
J(\omega)=\frac{1}{2 \pi} \int_{-\infty}^{\infty}|G(t)| e^{j \omega t} d t
$$

при $\delta<0$ имеет гауссову форму:

$$
J(\omega) \approx \frac{1}{\nu \sqrt{\pi \alpha}} e^{-\omega^{2} /\left(\nu^{2} \alpha\right)},
$$

где $\alpha=1$ при $T \gg T_{0}$ и $\alpha=T / T_{0}$ при $T \ll T_{0}, \nu=\sqrt{N / 2}|\delta| A_{\perp}$. В случае $\delta>0$ при $T \gg T_{0}$ также имеем гауссову форму линии: $J(\omega) \approx e^{-\omega^{2} / \nu^{2}} / \nu \sqrt{\pi}$. Интересная ситуация возникает в области $T \leqslant T_{0}$, где вблизи “критической" температуры $T_{0}$ 


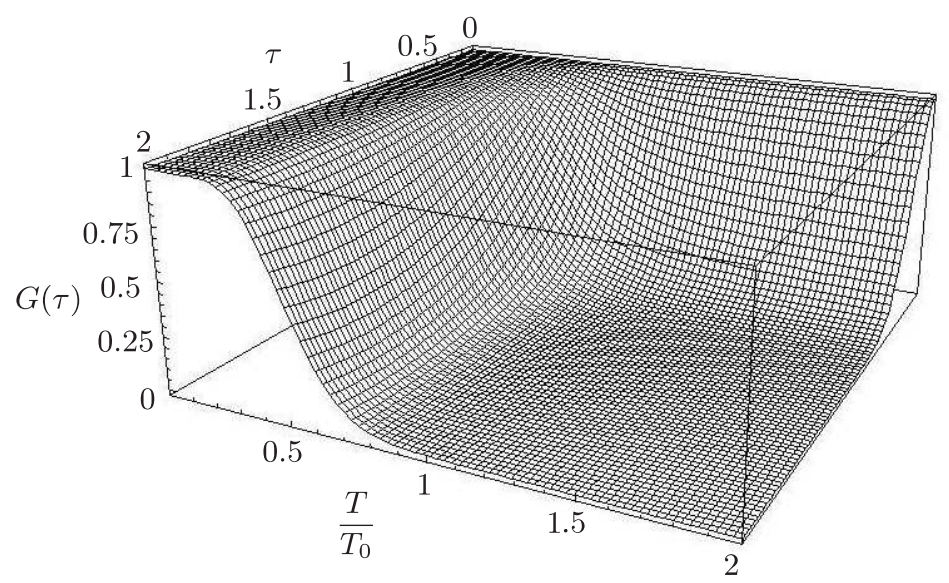

Рис. 2. Зависимость ССИ от времени и температуры в случае анизотропии “легкая ось" $(\delta>0)$.

линия ЯМР принимает вид

$$
\begin{aligned}
J(\omega) & \approx \frac{1}{\pi} \int_{0}^{\infty} \frac{\cos \omega t}{1+\delta^{2} A_{\perp}^{2} t^{2} /\left(4 \kappa^{2}\right)} d t=\frac{2 \kappa}{n|\delta| A_{\perp}} K_{0}\left(\frac{2 \kappa \omega}{|\delta| A_{\perp}}\right)= \\
& = \begin{cases}\frac{2 \kappa}{|\delta| A_{\perp}}\left(\ln \left(\frac{|\delta| A_{\perp}}{2 \kappa \omega}\right)+O(\omega)\right), & \omega \rightarrow 0, \\
\frac{\sqrt{\kappa}}{\sqrt{\pi|\delta| A_{\perp}}} \frac{1}{\sqrt{\omega}} e^{-2 \kappa \omega /\left(|\delta| A_{\perp}\right)}, & \omega \rightarrow \infty .\end{cases}
\end{aligned}
$$

Таким образом, вблизи температуры $T_{0}$ линия ЯМР содержит логарифмическую сингулярность при $\omega \rightarrow 0$ и экспоненциальный спад на концах. Этот функциональный вид линии отличается от традиционных гауссовых или лоренцевых кривых в теории неоднородного уширения [18]. На рис. 3, где показана зависимость формы линии спиновой системы от частоты и температуры при $\delta>0$, отчетливо видно появление сингулярности при $T=T_{0}$.

Важными характеристиками формы линии являются ее моменты [19]. В частности, момент второго порядка $M_{2}=-G_{t t}^{\prime \prime}(0)$ характеризует ширину линии, а отношение $M_{4} / M_{2}^{2}=G_{t^{4}}^{(4)}(0) /\left(G_{t t}^{\prime \prime}(0)\right)^{2}\left(M_{4}\right.$ - момент четвертого порядка) характеризует близость линии к традиционным гауссовой или лоренцевой формам. Для рассматриваемой модели при анизотропии “легкая плоскость" $(\delta<0)$ второй момент равен

$$
M_{2}=-G_{t t}^{\prime \prime}(0)=k^{2}\left(\frac{N}{1+T_{0} / T}+\frac{T_{0} / T}{\left(1+T_{0} / T\right)^{2}}\right) \approx \begin{cases}k^{2} N, & T \gg T_{0}, \\ \frac{T}{T_{0}} k^{2} N, & T \ll T_{0},\end{cases}
$$

где $k=|\delta| A_{\perp} / 2$, а значение отношения $M_{4} / M_{2}^{2}$ близко к 3 :

$$
\frac{M_{4}}{M_{2}^{2}}=\frac{G_{t^{4}}^{(4)}(0)}{\left(G_{t t}^{\prime \prime}(0)\right)^{2}}=3+\frac{6}{\left(1+T\left(1+T_{0} / T\right)^{2} N / T_{0}\right)^{2}} \approx 3
$$




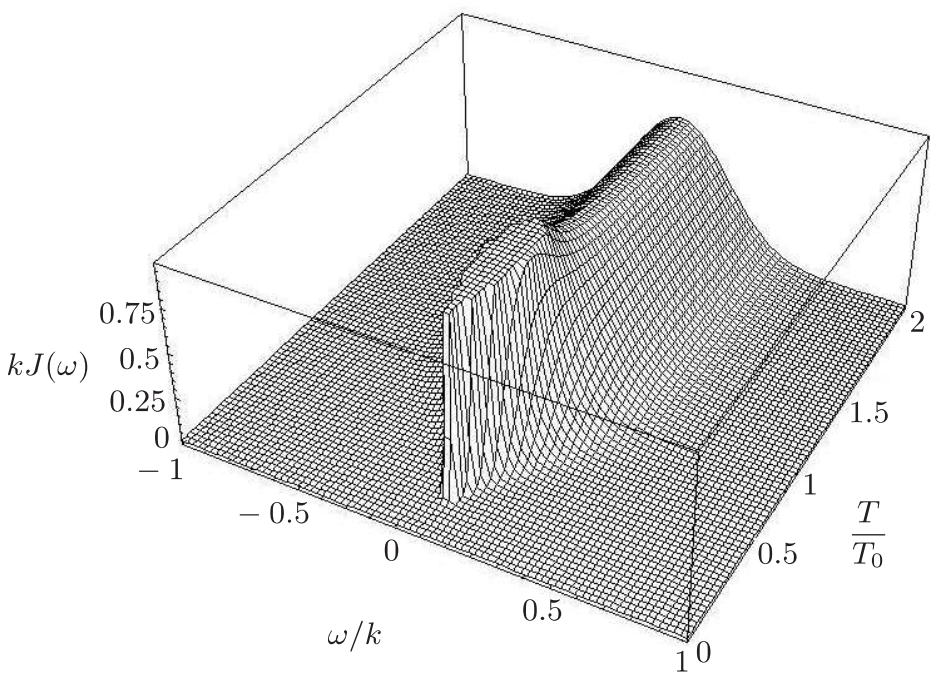

Рис. 3. Зависимость формы линии ЯМР от частоты и температуры при низотропии “легкая ось", $k=A_{\perp} \delta / 2$.

что указывает на гауссов тип линии [19]. В случае анизотропии “легкая ось" $(\delta>0)$ в области температур $T \leqslant T_{0}$ второй момент $M_{2}$ и отношение $M_{4} / M_{2}^{2}$ соответственно равны

$$
\begin{gathered}
M_{2}=-G_{t t}^{\prime \prime}(0)=k^{2}(\varphi(k)+N \psi(k)) \approx k^{2}\left(\frac{1}{k^{2}}+N\right), \\
\frac{M_{4}}{M_{2}^{2}}=\frac{G_{t^{4}}^{(4)}(0)}{\left(G_{t t}^{\prime \prime}(0)\right)^{2}}=3+6\left(\frac{\varphi(k)}{\varphi(k)+N \psi(k)}\right)^{2} \approx 9-12 N k^{2} .
\end{gathered}
$$

Из этих формул видно, что переход в область температур $T \leqslant T_{0}$ приводит к уширению линии, которая имеет форму, отличную от гауссовой $\left(M_{4} / M_{2}^{2} \approx 3\right)$ или лоренцевой $\left(M_{4} / M_{2}^{2} \gg 1\right)$. Результаты вычислений второго и четвертого моментов резонансной линии подтверждают выводы о форме линии, сделанные выше.

\section{4. ЗАКЛЮЧЕНИЕ}

В заключение приведем основные выводы.

1. Если в системе ядерных спинов, заключенных в потенциальную яму (наноконтейнер) имеется малый адиабатический параметр, который определяется тем, что несущие спины молекулы находятся в быстром движении, а их суммарный спин в свою очередь определяет медленную спиновую релаксацию индивидуальных спинов ядер под влиянием слабых спин-спиновых взаимодействий, то спин-спиновые взаимодействия усредняются, но не полностью. Остаточные (усредненные) спин-спиновые взаимодействия описываются двумя константами взаимодействия для всех пар спинов. В результате появляется возможность строгого аналитического описания формы линии ЯМР спиновой системы в наноконтейнере. 
2. Найденные выражения для ССИ и формы линии ЯМР для низких температур позволяют произвести сопоставление теоретических расчетов с экспериментальными данными с целью проверки предсказанных специфических термодинамических свойств рассматриваемой спиновой системы, в том числе наличие упорядоченных спиновых конфигураций и температурный интервал их проявления.

3. Специфическое термодинамическое поведение системы ядерных спинов в наноконтейнере при низких температурах, а именно образование топологических возбуждений (солитонов) и как следствие аномальное поведение теплоемкости, приводит к появлению логарифмической сингулярности в поведении линии ЯМР. Вычисленные параметры ССИ и формы линии позволяют количественно оценить температурный интервал влияния солитонов, а также величину спиновой щели в спектре магнитных возбуждений.

Отметим также, что возможность аналитического расчета спектров ЯМР рассмотренной спиновой системы позволяет получать информацию о средних размерах, форме и вариациях объема наноконтейнеров.

\section{Список литературы}

[1] А. Абрагам, Ядерный магнетизм, ИЛ, М., 1969.

[2] Ч. Сликтер, Основы теории магнитного резонанса, Пер. с англ., Изд. 2-е, Мир, М., 1981.

[3] А. Г. Лундин, Э. И. Федин, ЯМР-спектроскопия, Наука, М., 1986.

[4] В. Г. Показаньев, Г. В. Скроцкий, Л. И. Якуб, УФН, 116:7 (1975), 485-515.

[5] В. М. Дубовик, И. В. Лунегов, М. А. Марценюк, ЭЧАЯ, 26:1 (1995), 72-145.

[6] А.Р. Кессель, Р. Р. Нигматуллин, А. А. Хамзин, Н. А. Яковлева, ТМФ, 145:3 (2005), $411-419$.

[7] А. А. Хамзин, Р. Р. Нигматуллин, ТМФ, 165:1 (2010), 160-176.

[8] А. А. Хамзин, Р. Р. Нигматуллин, ЖЭЭФФ, 138:6 (2010), 1163-1174.

[9] J. Baugh, A. Kleinhammes, D. Han, Q. Wang, Y. Wu, Science, 294:5546 (2001), 1505-1508.

[10] E. B. Fel'dman, M. G. Rudavets, ЖЭЭТФ, 125:2 (2004), 233-246.

[11] М. Г. Рудавец, Э. Б. Фельдман, Писъма в ЖЭЭТФ, 75:12 (2002), 760-762.

[12] E. B. Fel'dman, M. G. Rudavets, Chem. Phys. Lett., 396:4-6 (2004), 458-464, arXiv: quant-ph/0409076.

[13] А. Абрагам, М. Гольдман, Ядерный магнетизм: порядок и беспорядок, Мир, М., 1984.

[14] М. Гольдман, Спиновая температура и ЯМР в твердых телах, Мир, М., 1972.

[15] М.В. Федорюк, Асимптотика: Интеграль и рядь, Наука, М., 1987.

[16] C. Scharf, Phys. Lett. A, 38:2 (1972), 123-124.

[17] Б. В. Мощинский, В.К. Федянин, ТМФ, 31:1 (1977), 101-106.

[18] R. Kubo, "A Stochastic Theory of Line Shape", Stochastic Processes in Chemical Physics, Adv. Chem. Phys., 15, ed. K. E. Shuler, John Wiley \& Sons, Inc., Hoboken, NJ, USA, 1969, 101.

[19] С. А. Альшуллер, Б. М. Козырев, Электронный парамагнитный резонанс соединений элементов промежуточных групп, Наука, М., 1972.

Поступила в редакцию 3.09.2010 